\title{
Incidencia del hormigón ligero y flexible en la optimizacion de secciones, ductilidad y desempeño estructural
}

\section{Incidence of the light and flexible concrete in the optimization of sections, ductility and structural performance}

Morales Luis Wladimir Universidad Central del Ecuador lwmorales@uce.edu.ec

Ruiz Alex Universidad Central del Ecuador

Revista Cumbres Vol.6 №1

Versión electrónica ISSN 1390-3365 http://investigacion.utmachala.edu.ec/revistas/index.php/Cumbres 


\title{
RESUMEN
}

El Ecuador es un país de alta peligrosidad sísmica, por ello es primordial conseguir que los elementos estructurales de las edificaciones sean dúctiles frente a las fuerzas laterales y gravitacionales, a la vez, que tengan la capacidad de disipar la energía de deformación inelástica, evitando el colapso y mejorando el desempeño estructural.

Para conseguir este propósito, se diseñó y fabricó un hormigón estructural con EPS de alta densidad en reemplazo del agregado grueso (métodos de Füller y Bolomey), además, se incluyó híper plastificante y fibras sintéticas de polipropileno, para obtener un hormigón flexible, ligero y estructural (HFLE) para posteriormente determinar las propiedades físico-mecánicas del hormigón como: $\gamma_{c^{\prime}} \mathrm{f}^{\prime} \mathrm{c}, \mathrm{Ec}, \mathrm{f}_{\mathrm{cr}} \mathrm{y} \varepsilon_{\mathrm{cu}}$.

Con los resultados, se demostró que la sección transversal de la viga con HFLE, reduce su sección en un $28 \%$ frente a una viga construida con HC, sin perder las características estructurales pues el HFLE posee un f'c a los 28 días de $37.43(\mathrm{MPa})$ mayor a $21(\mathrm{MPa})$ establecido en la NEC, y una $\varepsilon_{\mathrm{cu}}$ de $0.0058 \mathrm{~mm} / \mathrm{mm}$, resultando mayor a la $\varepsilon_{\mathrm{cu}}=0.003 \mathrm{~mm} / \mathrm{mm}$ establecida por el ACI 318-19; además, se obtuvo una densidad de 1898.68 kg/m3 menor en 17\% en comparación con el HC. Finalmente, se demostró que el uso del HFLE en edificaciones permite aumentar la capacidad resistente frente a la acción sísmica, mejora el desempeño de las estructuras, reduce el peso, disminuye las secciones de los elementos estructurales mejorando la concepción arquitectónica, proporciona mayor ductilidad y disipa de mejor manera la energía de deformación inelástica.

Palabras clave: Hormigón flexible, hormigón ligero, deformación unitaria, poliestireno, polipropileno, módulo de elasticidad.

\begin{abstract}
Ecuador is a country of high seismic danger. Therefore, it is essential to ensure that the structural elements of buildings are ductile against lateral and gravitational forces. At the same time, they must have the ability to dissipate inelastic deformation energy to avoid collapse and improve structural performance. To achieve this purpose, structural concrete with high density EPS was manufactured in replacement of the coarse aggregate (Füller and Bolomey methods). Hyper plasticizer and synthetic polypropylene fibers were included to obtain flexible, lightweight and structural concrete (HFLE). The physical-mechanical properties of concrete such as $\gamma_{\mathrm{c}^{\prime}} \mathrm{f}^{\prime} \mathrm{c}, \mathrm{Ec}, \mathrm{f}_{\mathrm{cr}}$ y and $\varepsilon_{\mathrm{cu}}$ were later determined. The results show that the cross section of the beam with HFLE reduces its section by $28 \%$ compared to a beam built with HC, without losing the structural characteristics. In 28 days, the HFLE has a $\mathrm{f}^{\prime} \mathrm{c}$ of $37.43(\mathrm{MPa})$ greater than $21(\mathrm{MPa})$ established in the NEC, and a $\varepsilon_{\text {си }}$ of $0.0058 \mathrm{~mm} / \mathrm{mm}$. It results greater than the $\varepsilon_{\mathrm{cu}}=0.003 \mathrm{~mm} / \mathrm{mm}$ established
\end{abstract}


by the ACI 318-19. In addition, a density of $1898.68 \mathrm{~kg} / \mathrm{m} 3$ lower by $17 \%$ was obtained compared to HC. Finally, it was demonstrated that the use of HFLE in buildings allows to increase the resistant capacity against seismic action. It also improves the performance of the structures, reduces the weight, decreases the sections of the structural elements, provides greater ductility, and dissipates better inelastic deformation energy.

Keywords: Flexible concrete, lightweight concrete, unit deformation, polystyrene, polypropylene, elastic modulus.

\section{INTRODUCCIÓN}

El hormigón es el principal material de construcción en obras civiles del país. Se debe realizar un hormigón capaz de soportar las cargas a la compresión, tracción, flexión y torsión que supere las de uno convencional, además nos ayude a reducir el peso total de la estructura con la finalidad de reducir la fuerza lateral producida por las fuerzas sísmicas. El Ecuador es un país de alta peligrosidad sísmica por efectos de subducción y actividad de fallas geológicas locales como la ciudad de Quito. Por ello, es primordial conseguir que los elementos estructurales de las edificaciones sean dúctiles frente a las fuerzas laterales y gravitacionales, y consiga mejorar la capacidad de disipar la energía de deformación inelástica, evitando el colapso y mejorando el desempeño estructural.

Basándose en este criterio, se diseñó y fabricó un hormigón HFLE, se determinó la deformación unitaria $(\mathcal{E} \mathrm{cu})$ del hormigón elaborado con poliestireno de alta densidad (EPS) en reemplazo del agregado grueso natural. Científicos de la Universidad Tecnológica de Nanyang, desarrollaron un hormigón flexible llamada ConFlexPave introduciendo microfibras de polímero en la mezcla de hormigón, trabajando en el NTU-JTC Industrial Infrastructure Innovation Centre (Lynch, 2016).

\section{MATERIALES}

Los materiales utilizados en esta investigación son los agregados de las canteras de San Antonio y Pifo, ubicadas dentro de la provincia de Pichincha, también se utilizó el cemento Chimborazo HE, fibras sintéticas de polipropileno, además se incluyó híper plastificante. La dosificación de una mezcla de hormigón esta función de los materiales que se van a utilizar y de la obra o investigación que se ha de construir con esa mezcla (Coro, 2014).

\section{Agregado grueso y fino}

Para la presente investigación, en las mezclas realizadas se utilizó agregado grueso de tamaño nominal máximo $3 / 4$ de pulgada, con superficie angular. También, se utilizaron agregados finos provenientes de las canteras de San 
Antonio y Pifo, con módulos de finura de 2.57 y 3.51, respectivamente. Las granulometrías de estos agregados se muestran en la Figura 1, Figura 2 y Figura 3.

Figura 1. Granulometría del agregado grueso (Pifo).

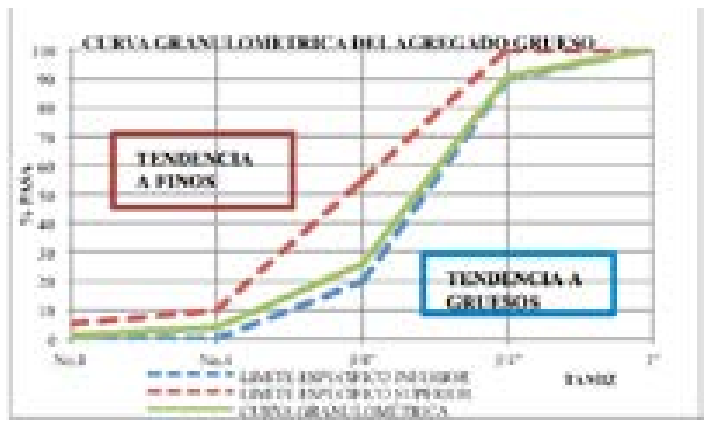

Figura 2. Granulometría del agregado fino (San Antonio).

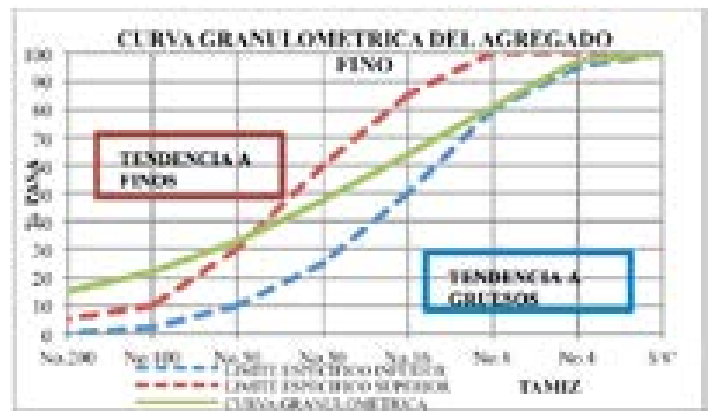

Figura 3. Granulometría del agregado fino (Pifo).

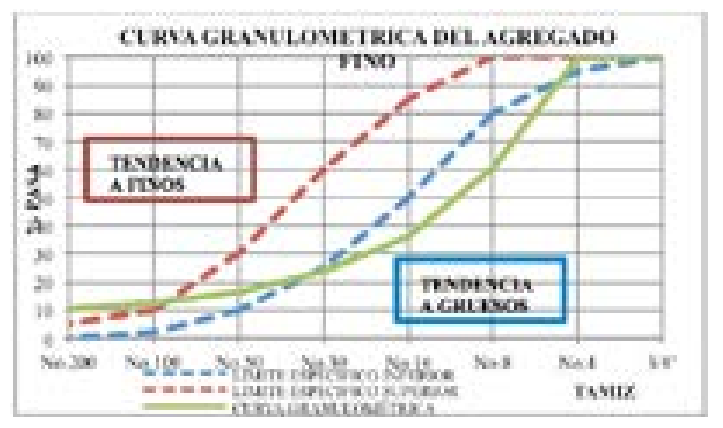

\section{Cemento}

Se utilizó el Cemento Chimborazo hidráulico de alta resistencia inicial (tipo HE), que cumple estrictamente la norma INEN 2380, (...). Este material es producido con Clinker, adiciones minerales, sulfato de calcio, estos componentes son dosificados en la molienda obteniendo un producto de alta fineza y calidad. Para asegurar la calidad mantienen un sistema de gestión de calidad basado en la Norma ISO 9001 (Cemento Chimborazo, 2018). La densidad de este cemento es $2.92 \mathrm{~g} / \mathrm{cm}^{3}$, resultado de varios ensayos de laboratorio.

\section{Cumbres}




\section{Componentes incorporados en el hormigón HFLE}

Fibra Sintética: Se utilizaron las macro fibras sintéticas de Ferromix, y han sido específicamente diseñadas para proveer el máximo beneficio en un amplio número de aplicaciones. Desde la reducción de las grietas por contracción plástica, cambios de temperatura, hasta remplazo de armadura secundaria. La dosificación recomendada por el fabricante es $2.0 \mathrm{~kg} / \mathrm{m}^{3}$ de hormigón para cementos y agregados del Ecuador.

Aditivo plastificante: Estos aditivos tienen la capacidad de reducir el agua de mezclado hasta porcentajes superiores al 30\%, ayuda a mejorar las propiedades reológicas y mecánicas del concreto, como son la trabajabilidad, módulo de elasticidad, resistencia a la compresión y flexión.

Poliestireno expandible (EPS): Es un plástico que se genera de la expansión del poliestireno "STYROPOR BF 327" de la casa BASF, su característica es ser inifugo, antillama y autoextinguible; no contiene compuestos cloroflourcarbonatos. Se empleó este material ligero para reducir el peso del hormigón.

\section{Agua de mezclado}

El agua utilizada fue la proporcionada por el sistema de agua potable de la ciudad de Quito, según la norma NTE INEN 2617: 2012, donde se indica que, "se permite el uso del agua potable como agua de mezcla del hormigón sin la realización de ensayos para verificar su cumplimiento de los requisitos de esta norma" (NTE INEN 2617, 2012).

\section{MÉTODOS}

Los métodos utilizados para determinar las características de los agregados en la presente investigación fueron los establecidos en las normas ASTM y NEC, los cuales fueron el punto de partida para poder obtener resultados válidos y reales. La caracterización de materiales se determinó según las normas INEN, normas ASTM, las mismas que se detallan en la Tabla 1, ACI 211, ACI 318.

Para conseguir una mezcla optima se realizó el diseño según la norma (ACI 211.4R, 2008), además una combinación de agregados utilizando los métodos de Füller - Thompson, y Bolomey se basan en seleccionaron una curva granulométrica continua para la composición óptima de los agregados en el concreto (Giraldo, 1987). Se procedió a reemplazar el 25\% del de árido grueso por EPS de alta densidad, además complementarlo con la utilización de macro fibra sintética con el propósito de conseguir un hormigón flexible, ligero y estructural.

Tabla 1. Normas utilizadas en la investigación para ensayos de los agregados. 


\begin{tabular}{|c|c|c|}
\hline ENSAYO & INEN & ASTM \\
\hline -Abrasión & INEN 860 & ASTM C-131 \\
\hline -Colorimetría & INEN 855 & ASTM C-040 \\
\hline $\begin{array}{l}\text {-Densidad real especifico } \\
\text { Agregado fino } \\
\text { Agregado grueso }\end{array}$ & $\begin{array}{l}\text { INEN } 856 \\
\text { INEN } 857\end{array}$ & $\begin{array}{l}\text { ASTM C-127 } \\
\text { ASTM C-128 }\end{array}$ \\
\hline -Capacidad de absorción & $\begin{array}{l}\text { INEN } 856 \\
\text { INEN } 857\end{array}$ & $\begin{array}{l}\text { ASTM C-070 } \\
\text { ASTM C-070 }\end{array}$ \\
\hline -Contenido de humedad de los agregados & INEN 862 & ASTM C-566 \\
\hline -Densidad aparente suelta y compactada & INEN 858 & ASTM C-029 \\
\hline $\begin{array}{l}\text {-Granulometría } \\
\text { Agregado fino } \\
\text { Agregado grueso }\end{array}$ & $\begin{array}{l}\text { INEN } 696 \\
\text { INEN } 696\end{array}$ & $\begin{array}{l}\text { ASTM C-136; ASTM C-033 } \\
\text { ASTM C-125 }\end{array}$ \\
\hline
\end{tabular}

La presente investigación se enfoca en dos requerimientos principales: el primero es conseguir un hormigón ligero, es decir, con una densidad en estado seco al aire menor a $1920 \mathrm{~kg} / \mathrm{m}^{3}$ según ACI 213R (2003), y según la norma NTE INEN 1762 (2015) una densidad menor a 2000 kg/m3 ; el segundo requerimiento es determinar la deformación unitaria de un hormigón flexible, esto es, una deformación superior a la especificada en el ACI 318 (2014), que para hormigones convencionales se adopta un valor de $0.003 \mathrm{~mm} / \mathrm{mm}$.

\section{Dosificación inicial}

Para el diseño del hormigón, se siguieron las recomendaciones de dosificación dadas por el comité ACI 211, los métodos de Füller - Thompson, y Bolomey, además se considera una resistencia específica a la compresión del hormigón de f'c $=40 \mathrm{MPa}$ a los 28 días de edad. La resistencia requerida se determinó de acuerdo a los procedimientos proporcionados por los comités del ACI 211.4R y ACI 318, siendo de $49 \mathrm{MPa}$ en los dos casos, se obtuvo por la mezcla con un $\mathrm{f}^{\prime} \mathrm{c}=49.01 \mathrm{MPa}$ a los 28 días. La Tabla 2 muestra la dosificación inicial para 1m3 de hormigón, utilizada para las distintas variaciones de agregado grueso por EPS. En la Tabla 3 se evidencia los resultados a compresión simple del hormigón con la dosificación inicial (hormigón convencional) a los 7, 14, 28 días de edad.

Tabla 2. Dosificación de la mezcla patrón definitiva

Tabla 3. Ensayo a compresión simple del hormigón convencional

\begin{tabular}{|l|l|}
\hline DOSIFICACIÓN DE LA MEZCLA PATRÓN DEFINITIVA \\
\hline Cemento & 1 \\
\hline A grueso (PIFO) & 1.84 \\
\hline A fino (SA) & 1.70 \\
\hline A fino (PIFO) & 0.73 \\
\hline Agua & 0.50 \\
\hline
\end{tabular}




\begin{tabular}{|l|l|l|l|}
\hline MUESTRA & EDAD & \multicolumn{3}{|l|}{ RESISTENCIA } \\
\cline { 3 - 5 } & (DİAS) & $\mathrm{MPa}$ & $\%$ \\
\hline \multirow{3}{*}{$\mathrm{AC}$} & 7 & 39.35 & 80.30 \\
\cline { 2 - 5 } & 14 & 44.07 & 89.93 \\
\cline { 2 - 5 } & 28 & 49.01 & 100.01 \\
\hline
\end{tabular}

\section{RESULTADOS}

\section{Densidad especifica}

Los valores de las densidades calculadas según la norma NTE INEN 1573, cuyos resultados de los diferentes tipos de hormigones se muestran en la Tabla 5, como se puede observar la mezcla de hormigón HFLE según las especificaciones de norma NTE INEN 1762 (2015), se establece que el hormigón es ligero con un valor de $1898.68 \mathrm{~kg} / \mathrm{cm}^{3}$, disminuyendo su densidad en un 17.07\% en comparación a la del Hormigón Convencional.

\section{Resistencia a la compresión}

Las resistencias obtenidas en las pruebas a compresión a la edad de 28 días, conforme a la norma NTE INEN 1573 (2010) cuyos resultados se muestran en la Tabla 7. Como puede observarse, la mezcla de hormigón HFLE dio como resultado una resistencia última a la compresión f“" c de $37.43 \mathrm{MPa}$, considerado un hormigón estructural al cumplir con el parámetro establecido en la norma NEC-SE-HM.

\section{Deformación Unitaria}

Según el método establecido en la norma ASTM C469/C469M (2014) para determinar el módulo de elasticidad estático del concreto sometido a compresión, cuyos valores se observan en la Tabla 8, dando como resultado para el Hormigón + Aditivo + Fibras + 25\% EPS un valor igual a 0.0058 mm/ mm mayor al de un hormigón convencional, se establece que se trata de un hormigón flexible al tener una mayor capacidad de la máxima deformación unitaria utilizable en la hipótesis de diseño según la norma NEC-SE-HM.

\section{Flexión en Vigas}

La determinación del esfuerzo a flexión de vigas simples con carga en el punto medio se estableció según la norma (ASTM C 293, 2002), donde los resultados de los ensayos se resumen en la Tabla 9, las vigas de hormigón HFLE con los siguientes espesores $150(\mathrm{~mm}) ; 50(\mathrm{~mm})$ y $20(\mathrm{~mm})$ alcanzaron módulos de rotura de $74.47\left(\mathrm{~kg} / \mathrm{cm}^{2}\right) ; 61.20\left(\mathrm{~kg} / \mathrm{cm}^{2}\right) ; 76.88\left(\mathrm{~kg} / \mathrm{cm}^{2}\right)$ respectivamente, estos valores superan el módulo de rotura teórico calculado 
según lo establecido en la norma (ACI 318, 2014), obteniendo un valor de $38.69\left(\mathrm{~kg} / \mathrm{cm}^{2}\right)$.

\section{Diseño de viga a flexión con HC y HFLE}

Se realizó el diseño de dos vigas a flexión una con un hormigón convencional y la otra con el hormigón HFLE, el detalle de la viga se observa en la Figura 4, utilizando los parámetros de la norma ACI 318 y la NEC-SE-HM, los resultados obtenidos se detallan en la Tabla 4, y la comprobación del diseño se resume en la Tabla 5. Como resultado se distinguió un aumento de los requerimientos de diseño, con la finalidad de reducir la sección de la viga. En la Figura 7 y Figura 8 se indican los detalles de la armadura a flexión de las vigas.

Figura 4. Viga a diseñar

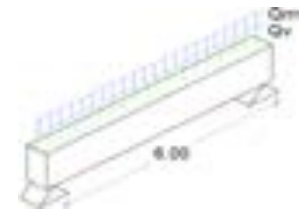

\section{Código de Diseño (ACI 318-19)}

$\varepsilon_{\mathrm{t}}=0.005$

$\boldsymbol{\varnothing}=0.9$

Diseño de una viga con hormigón convencional

\section{Datos:}

$\mathrm{f}^{\prime} \mathrm{C}=240 \mathrm{~kg} / \mathrm{cm}^{2}$

$\mathrm{fy}=4200 \mathrm{~kg} / \mathrm{cm}^{2}$

Luz viga $=6 \mathrm{~m}$

qc.muerta $=5.2 \mathrm{t} / \mathrm{m}$

qc.viva $=2.0 \mathrm{t} / \mathrm{m}$

$\varepsilon_{\mathrm{cu}}=0.003 \mathrm{~m} / \mathrm{m}$

$\gamma_{\text {hor }}=2.4 \mathrm{t} / \mathrm{m}^{3}$

$\beta_{1}=0.85$

*No incluye peso propio de la viga

Diseño de una viga con hormigón + aditivo + fibra + $25 \%$ EPS

\section{Datos:}

$$
\begin{aligned}
& \mathrm{f}^{\prime} \mathrm{c}=37.43 \mathrm{MPa}=381.68 \mathrm{~kg} / \mathrm{cm}^{2} \\
& \text { fy }=4200 \mathrm{~kg} / \mathrm{cm}^{2} \\
& \text { Luz viga }=6 \mathrm{~m} \\
& \mathrm{qcm}=5.2 \mathrm{t} / \mathrm{m} \\
& \mathrm{qcv}=2.0 \mathrm{t} / \mathrm{m} \\
& \varepsilon_{\mathrm{cu}}=0.0058 \mathrm{~m} / \mathrm{m} \\
& \gamma_{\mathrm{hor}}=1.90 \mathrm{t} / \mathrm{m}^{3} \\
& \beta_{1}=0.78 \\
& \beta 1=0.85-\frac{0.05\left(f^{\prime} c(M P a)-28\right)}{7}
\end{aligned}
$$

*No incluye peso propio de la viga

Figura 5. Diagramas de momento y corte (incluye peso propio de la viga). Hormigón convencional. Figura 6. Diagramas de momento y corte (incluye peso propio de la viga). HFLE.

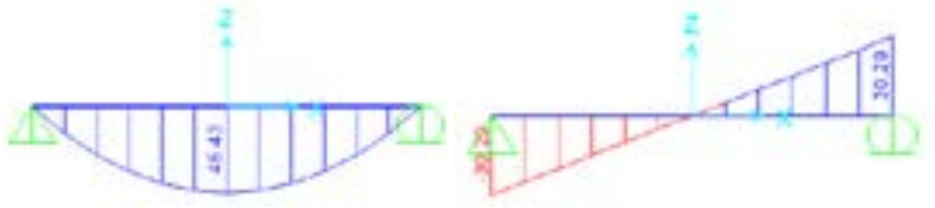

Figura 7. Esquema de armadura a flexión en viga de hormigón convencional. 
Figura 8. Esquema de armadura a flexión en viga de HFLE.
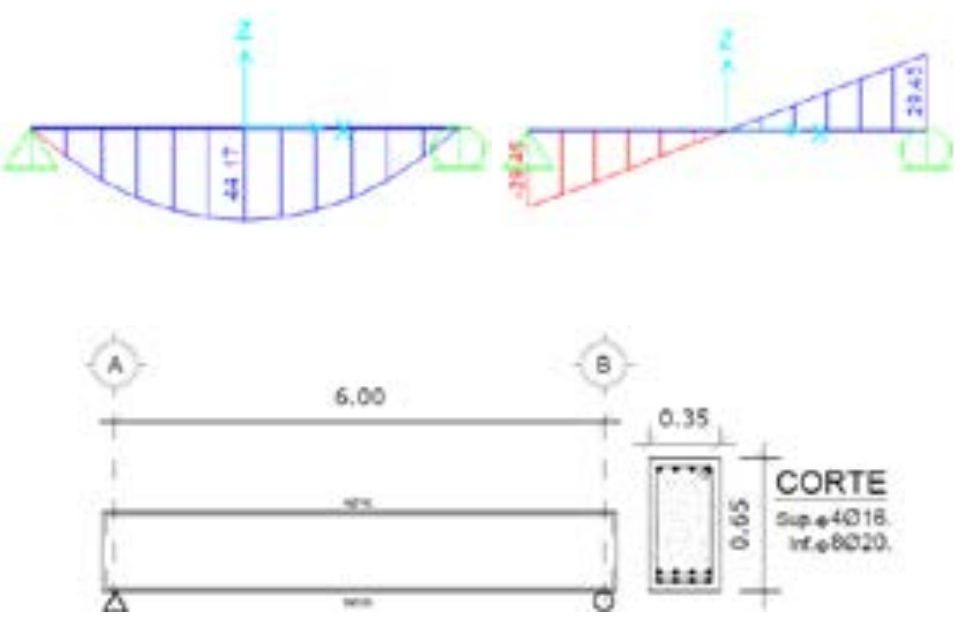

Tabla 4. Resultados de diseño a flexión

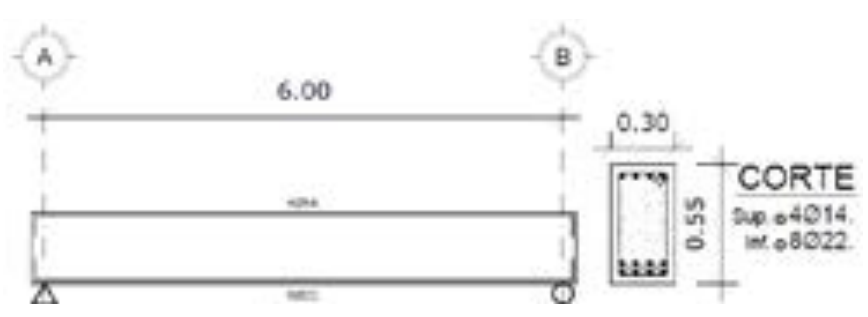

Tabla 5. Comprobación del diseño de vigas

Tabla 6. Densidad específica

\begin{tabular}{|c|c|c|c|c|c|c|c|}
\hline MEZCLA & P_MAX & $\mathbf{W}$ & $\begin{array}{l}\text { RU } \\
\left(\mathrm{KG} / \mathrm{CM}^{2}\right)\end{array}$ & $\begin{array}{l}\text { B } \\
\text { (CM) }\end{array}$ & $\begin{array}{l}\text { D } \\
(\mathrm{CM})\end{array}$ & $\begin{array}{l}\mathbf{H} \\
\text { (CM) }\end{array}$ & $\begin{array}{l}\mathrm{A} \\
\left(\mathrm{CM}^{2}\right)\end{array}$ \\
\hline \multirow[t]{3}{*}{ Hormigón Convencional } & \multirow{3}{*}{0.01548} & \multirow{3}{*}{0.27094} & \multirow{3}{*}{54.631} & 30 & 63.67 & 70 & \multirow{3}{*}{2275} \\
\hline & & & & 35 & 57.00 & 65 & \\
\hline & & & & 40 & 55.48 & 60 & \\
\hline \multirow{3}{*}{$\begin{array}{l}\text { Hormigón +Aditivo + Fibras } \\
+25 \% \text { EPS }\end{array}$} & \multirow{3}{*}{0.03013} & \multirow{3}{*}{0.33150} & \multirow{3}{*}{101.780} & 30 & 46.70 & 55 & \multirow{3}{*}{1650} \\
\hline & & & & 35 & 45.40 & 50 & \\
\hline & & & & 40 & 44.05 & 45 & \\
\hline
\end{tabular}

Tabla 7. Resistencia a la compresión edad 28 días

\begin{tabular}{|l|l|l|r|r|r|r|}
\hline MEZCLA & P_CALC & $\begin{array}{l}\text { AS } \\
\left(\mathbf{C M}^{2}\right)\end{array}$ & $\begin{array}{l}\boldsymbol{\varepsilon} \mathbf{0} \geq \\
\mathbf{0 . 0 0 5}\end{array}$ & $\begin{array}{l}\text { ASMIN } \\
\left(\mathbf{C M}^{2}\right)\end{array}$ & $\begin{array}{l}\text { MU } \\
(\mathbf{T}-\mathbf{M})\end{array}$ & $\begin{array}{l}\boldsymbol{\Phi} \mathbf{\text { MN }} \\
(\mathbf{T}-\mathbf{M})\end{array}$ \\
\hline Hormigón Convencional & 0.01207 & 24.08 & 0.00577 & 6.65 & 45.43 & 52.27 \\
\hline $\begin{array}{l}\text { Hormigón +Aditivo + } \\
\text { Fibras + 25\%EPS }\end{array}$ & 0.02065 & 28.93 & 0.00672 & 4.67 & 44.17 & 46.14 \\
\hline
\end{tabular}

Tabla 8. Deformación unitaria

\begin{tabular}{|l|l|}
\hline MEZCLA & $\begin{array}{l}\text { DENSIDAD ESPECIFICA(A) } \\
\left(\mathbf{K G} / \mathbf{M}^{\mathbf{3}}\right)\end{array}$ \\
\hline Hormigón Convencional & 2289.59 \\
\hline Hormigón +Aditivo + Fibras & 2301.71 \\
\hline Hormigón +Aditivo + Fibras + 25\%EPS & 1898.68 \\
\hline
\end{tabular}


Tabla 9. Módulo de rotura

\begin{tabular}{|c|c|}
\hline MEZCLA & $\begin{array}{l}\text { RESISTENCIA A LA COMPRESIÓN } \\
\text { (F'C) } \\
\text { (MPA) }\end{array}$ \\
\hline Hormigón Convencional & 49.01 \\
\hline Hormigón +Aditivo + Fibras & 59.64 \\
\hline Hormigón +Aditivo + Fibras + 25\%EPS & 37.43 \\
\hline
\end{tabular}

\begin{tabular}{|c|c|}
\hline MEZCLA & $\begin{array}{c}\text { DEFORMACIÓN UNITARIA } \\
\text { (ECU) } \\
\text { (MM/MM) }\end{array}$ \\
\hline Hormigón Convencional & 0.0039 \\
\hline Hormigón +Aditivo + Fibras & 0.0044 \\
\hline Hormigón +Aditivo + Fibras + & 0.0058 \\
\hline 25\%EPS & \\
\hline
\end{tabular}

\begin{tabular}{|c|c|c|c|}
\hline \multirow[t]{2}{*}{ MEZCLA } & \multicolumn{3}{|c|}{$\begin{array}{l}\text { MÓDULO DE ROTURA (MR) } \\
\left(\mathrm{KG} / \mathrm{CM}^{2}\right)\end{array}$} \\
\hline & Espesor $150(\mathrm{~mm})$ & Espesor $50(\mathrm{~mm})$ & Espesor $20(\mathrm{~mm})$ \\
\hline Hormigón Convencional & 53.85 & 45.48 & 46.50 \\
\hline $\begin{array}{l}\text { Hormigón +Aditivo + } \\
\text { Fibras }\end{array}$ & 70.97 & 69.72 & 70.13 \\
\hline $\begin{array}{l}\text { Hormigón +Aditivo + } \\
\text { Fibras + 25\%EPS }\end{array}$ & 74.47 & 61.20 & 76.88 \\
\hline
\end{tabular}

\section{DISCUSIÓN DE RESULTADOS}

El procedimiento adoptado para obtener la dosificación óptima utilizando las curvas de Füller y Bolomey para la combinación de agregados de las canteras de Pifo y San Antonio, cemento Chimborazo HE, EPS, fibras de polímeros Ferromix, y aditivo Viscomix MC-AG, al comparar las características mecánicas del hormigón como: densidad especifica detallada en la Tabla 5, las resistencias obtenidas en la Tabla 7 y las deformación unitarias cuyos valores se muestran la Tabla 8, se establece que el hormigón HFLE es ligero, estructural y flexible, al cumplir los 3 parámetros por lo que se diseñaron vigas de distintos espesores para determinar el módulo de rotura detalla en la Tabla 9 y como se esperaba resultaron ser mayores a la de un hormigón convencional.

El diseño de la viga a flexión con hormigón HFLE dio como resultado una disminución del área de hormigón del elemento igual al 27.5\% en comparación a una viga convencional, como se aprecian los resultados en la Tabla 4. 


\section{CONCLUSIONES}

Se demostró que HFLE es un hormigón ligero al tener una densidad de $1898.68\left(\mathrm{~kg} / \mathrm{m}^{3}\right)$, sin perder la característica estructural al poseer una resistencia la compresión a los 28 días de 37.43 (MPa). Un hormigón ligero en edificaciones ayuda a reducir el peso total de la estructura y asi disminuir las fuerzas laterales provocado por el sismo al ser directamente proporcional al peso de las edificaciones.

Un hormigón convencional tiene el valor de la deformación unitaria de $0.0030(\mathrm{~mm} / \mathrm{mm})$, para el hormigón HFLE se obtuvo una deformación unitaria de $0.0058(\mathrm{~mm} / \mathrm{mm})$, este último es mayor en un $93 \%$ por lo tanto se considera que el hormigón es flexible, aportando en el diseño de vigas por consiguiente reduce las secciones e influye en las deflexiones, derivas y rigidez de una estructura para disminuir costos en la cantidad de hormigón utilizado en este tipo de elementos estructurales.

Su aplicación en la elaboración de mampostería contribuirá a la reducción del peso de las mismas, debido a su baja densidad y su buena resistencia para este tipo de elementos no estructurales, además de mejorar la capacidad térmica y acústica de las edificaciones.

\section{REFERENCIAS BIBLIOGRÁFICAS}

ACI 211.4R. (2008). Guide for Selecting Proportions for High-Strength Concrete Using Portland Cement and Other Cementitious Materials. Farmington Hills, U.S.A.

ACI 213R. (2003). Guide for Structural Lightweight-Aggregate Concrete. Farmington Hills, Míchigan, Estados Unidos.

ACI 318. (2014). Requisitos de reglamento para concreto estructural (ACI 318S-14). Farmington Hills, Míchigan, Estados Unidos.

Admix. (2016). Ferromix. Quito.

ASTM C 293. (2002). Standard Test Method for Flexural Strength of Concrete (Using Simple Beam With Center-Point Loading). West Conshohocken, Pensilvania, USA.

ASTM C469/C469M. (2014). Método estándar para la determinación del módulo de elasticidad estático y de la relación de Poisson del concreto sometido a compresión.

Cemento Chimborazo. (2018). Ficha técnica cemento hidráulico de alta resistencia inicial tipo HE. Quito: UCEM.

Coro, M. E. (2014). Determinación del módulo de rotura en vigas de hormigón, fabricado con materiales procedentes de la cantera San Roque, para $\mathrm{f}^{\prime} \mathrm{c}=28 \mathrm{MPa}$ (Tesis de pregrado). Universidad Central del Ecuador.

Giraldo, O. (1987). Guía práctica para el diseño de mezclas de hormigón. Medellín, Antioquia, Colombia. 
Harmsen, T. E. (2002). Diseño de estructuras de concreto armado. Perú.

Lynch, P. (2016, 09 15). Investigadores del NTU Singapore desarrollan un hormigón flexible que resiste el agrietamiento. Retrieved 06 02, 2018, from www.plataformaarquitectura.cl: https://www.plataformaarquitectura.cl/cl/795253/investigadores-del-ntu-singapore-desarrollan-un-hormigon-flexible-que-resiste-el-agrietamiento

Norma Ecuatoriana de la Construcción. (2015). NEC - SE - HM. Ecuador.

NTE INEN 1573. (2010). Hormigón de cemento hidráulico. Determinación de la resistencia a la compresión de especímenes cilíndricos de hormigón de cemento hidráulico. Quito.

NTE INEN 1762. (2015). Hormigón. Definiciones y terminollogía. Quito: INEN.

NTE INEN 2617. (2012). Hormigón de cemento hidráulico. Agua para Mezcla. Quito. 